Pacific Journal of Mathematic 


\section{COMPACT ENDOMORPHISMS OF BANACH ALGEBRAS}

\section{HERBERT KAMOWITZ}

Let $T$ be a compact endomorphism of a commutative semisimple Banach algebra $B$. This paper discusses the behavior of the adjoint $T^{*}$ of $T$ on the set $X^{\prime}$ of multiplicative linear functionals on $B$. In particular it is shown that $\cap T^{* n}\left(X^{\prime}\right)$ is finite, thus generalizing the example of compact endomorphisms of the disc algebra.

o. Introduction and preliminaries. In this paper we discuss maps which are simultaneously endomorphisms of Banach algebras and compact operators. That is, these operators $T$ are linear, satisfy $T(f g)=(T f)(T g)$ for all $f$ and $g$ in the algebra and map bounded sets into sequentially compact sets.

As a motivating example, consider the disc algebra $A$, the supnorm algebra of functions analytic on the open unit disc $D$ and continuous on $\bar{D}$. Every nonzero endomorphism $T$ of $A$ has the form $T f=f \circ \varphi$ for $f \in A$, where $\varphi \in A$ and $\varphi$ maps $\bar{D}$ into $\bar{D}$. It was shown in [3] that if $\varphi$ is not a constant function, then $T$ is compact if, and only if, $|\varphi(z)|<1$ for all $z \in \bar{D}$. Moreover, for such $\varphi$, if $\varphi_{n}$ denotes its $n$th iterate, then $\cap \varphi_{n}(\bar{D})=\left\{z_{0}\right\}$ for some $z_{0} \in D$, and further the spectrum $\sigma(T)$ of $T$ satisfies $\sigma(T)=\left\{\left(\varphi^{\prime}\left(z_{0}\right)\right)^{n} \mid n\right.$ is a positive integer $\} \cup\{0,1\}$. When $\varphi$ is a constant function, the range of $T$ is one-dimensional and $T$ is compact with $\sigma(T)=\{0,1\}$.

We will now consider compact endomorphisms of other Banach algebras and study to what extent the properties of compact endomorphisms of the disc algebra can be generalized. Our principal results will describe the behavior of the adjoint $T^{*}$ of $T$ on the maximal ideal space of the algebra.

We first introduce some notation and terminology. Let $B$ be a commutative semi-simple Banach algebra with unit 1 and maximal ideal space $X$ and, in addition, let $\theta$ denote the zero functional on $B$. If $0 \neq T$ is a (necessarily) bounded endomorphism of $B$, then the adjoint $T^{*}$ induces a continuous function $\varphi$ from $X^{\prime} \equiv X \cup\{\theta\}$ into itself in the following way. For $x \in X$, let $e_{x} \in B^{*}$ satisfy $e_{x}(f)=\hat{f}(x)$, where $f \rightarrow \hat{f}$ is the Gelfand transformation of $B$. It is easy to verify that $T^{*} e_{x}$ is multiplicative. There are two possibilities. If $T^{*} e_{x} \neq \theta$, then $T^{*} e_{x}=e_{y}$ for some $y \in X$ and we let $\varphi(x)=y$. For the second case, if $T^{*} e_{x}=\theta$, we let $\varphi(x)=\theta$. We also define $\varphi(\theta)=\theta$. Since $\varphi$ is essentially equal to $T^{*}$ restricted to the set of multiplicative linear functionals on $B, \varphi$ is a continuous function from $X^{\prime}$ to $X^{\prime} ; \varphi$ will be called the map on $X$ or $X^{\prime}$ 
induced by $T$.

It is useful to note that if $T 1=1$, then $T^{*} e_{x} \neq 0$ for all $x \in X$ since $\left(T^{*} e_{x}\right)(1)=e_{x}(T 1)=e_{x}(1)=1$. Consequently, when $T 1=1, \varnothing$ maps $X$ into $X$. On the other hand, if $T 1 \neq 1$, then $\varphi(x)=\theta$ for some $x \in X$.

If $n$ is a positive integer, we let $\phi_{n}$ denote the $n$th iterate of $\varphi$, i.e., $\varphi_{0}(x)=x$ and $\varphi_{n}(x)=\varphi\left(\varphi_{n-1}(x)\right)$ for $x \in X^{\prime}$. A routine topological argument shows that $\cap \varphi_{n}\left(X^{\prime}\right)$ is a nonempty compact subset of $X^{\prime}$ and $\cap \varphi_{n}\left(X^{\prime}\right)$ is mapped onto itself by $\varphi$. Further, when $X$ is connected and $T \neq 0$, then $T 1=1$, whence $\varphi$ maps $X$ into $X$, $\cap \varphi_{n}(X)$ is connected and $\varphi$ maps $\cap \varphi_{n}(X)$ onto itself.

In the first section we will prove some structure theorems leading to the following theorem.

THEOREM 1.7. Suppose $B$ is a commutative semi-simple Banach algebra with unit 1 and maximal ideal space $X$ and $T$ is a nonzero compact endomorphism of $B$. If $\varphi$ is the map on $X^{\prime}$ induced by $T$, then $\cap \varphi_{n}\left(X^{\prime}\right)$ is finite. If $X$ is connected, then $\cap \varphi_{n}(X)$ is a singleton.

We recall that we have already characterized the compact endomorphisms of the disc algebra. Moreover, it is easy to verify that for any commutative semi-simple Banach algebra with unit 1 and maximal ideal space $X$, and any $a \in X$, the endomorphism $T: f \rightarrow$ $\widehat{f}(a) 1$ is compact. Using Theorem 1.7 , we will prove that if $X$ is a compact connected Hausdorff space, then every nonzero compact endomorphism $T$ on $C(X)$ has the form $T f=f(a) 1$ for some $a \in X$. Finally we will discuss some relations between the range $\varphi(X)$ of the induced map $\varphi$ of a compact endomorphism and the strong and Silov boundaries of other function algebras on $X$.

1. We begin with the following lemma dealing with the spectral radius $\|T\|_{s p}$ of a compact endomorphism.

Lemma 1.1. Suppose $B$ is a commutative semi-simple Banach algebra with unit 1 . If $T$ is a compact endomorphism of $B$ and $T$ is not nilpotent, then $\|T\|_{s p}=1$.

Proof. If $B$ is semi-simple and $\lambda$ is an eigenvalue of any endomorphism $T$ of $B$, then for each positive integer $n, \lambda^{n}$ is also an eigenvalue. For, if $0 \neq f \in B$ and $T f=\lambda f$, then $T\left(f^{n}\right)=(T f)^{n}=$ $\lambda^{n} f^{n} \neq 0$. On the other hand, when $T$ is a compact operator, every nonzero element in the spectrum $\sigma(T)$ is an eigenvalue [4]. Since $\sigma(T)$ is a compact subset of the plane, it follows that if $T$ is a 
compact endomorphism of $B$, then $\sigma(T) \subset\{\lambda|| \lambda \mid \leqq 1\}$.

It is easy to see that an endomorphism $S$ of $B$ is zero if, and only if, $S 1=0$. Thus an endomorphism $T$ is nilpotent if, and only if, $T^{m} 1=0$ for some positive integer $m$. Assume $T$ is an endomorphism of $B$ which is not nilpotent and set $F_{m}=T^{m} 1$. Then for each $m, F_{m}$ is a nonzero idempotent in $B$ and so

$$
1=\left\|\hat{F}_{m}\right\|_{\infty}=\left\|\left(T^{m} 1\right)^{\wedge}\right\|_{\infty} \leqq\left\|T^{m} 1\right\|_{B} \leqq\left\|T^{m}\right\|\|1\| \text {. }
$$

Since this holds for all positive integers $m$, it follows that $1 \leqq$ $\lim _{m \rightarrow \infty}\left\|T^{m}\right\|^{1 / m}=\|T\|_{s p}$. Combining this with the first paragraph gives that if $T$ is a compact endomorphism of a commutative semisimple Banach algebra with unit, then $\|T\|_{s p}=1$ if, and only if, $T$ is not nilpotent.

REMARKS. (1) Every quasinilpotent compact endomorphism of a commutative semi-simple Banach algebra with unit is nilpotent.

(2) The hypothesis in Lemma 1.1 that $B$ be semi-simple was needed to indure that $0 \neq f \in B$ implied $0 \neq f^{n} \in B$ for every positive integer $n$.

(3) If $B$ is not assumed to be semi-simple, then any denumerable plane set $\sigma$ with zero as its only limit point can be the spectrum of a compact endomorphism of $B$. For, it is well known that for each such $\sigma$ there exists a compact linear operator $T$ on Hilbert space $H$ with $\sigma(T)=\sigma$. If multiplication is defined on $H$ by $f g=0$ for all $f, g \in H$, then $H$ is a commutative Banach algebra, $T$ is a compact endomorphism on $H$ and $\sigma(T)=\sigma$.

The proof of the next lemma is straightforward.

LEMMA 1.2. Let $B$ be a commutative semi-simple Banach algebra with unit 1 and maximal ideal space $X$. If $E$ is a nonzero idempotent in $B$, then $B E$ and $B(1-E)$ are closed subalgebras of $B$ with units $E$ and $1-E$, respectively, and $B=B E \oplus B(1-E)$. If $Z=\{x \in X \mid \hat{E}(x)=1\}$, then the maximal ideal spaces of $B E$ and $B(1-E)$ are $Z$ and $X \backslash Z$, respectively. Further, if $T$ is an endomorphism of $B$ with $T E=E$, then $B E$ and $B(1-E)$ are invariant under $T$ in the sense that $T: B E \rightarrow B E$ and $T: B(1-E) \rightarrow B(1-E)$.

Lemma 1.3. Assume $T$ is a nonzero compact endomorphism of a commutative semi-simple Banach algebra $B$ with unit 1 . Then there exists a smallest nonnegative integer $M$ such that $T^{M} 1=T^{M+1} 1$. If $T$ is not nilpotent, then $E=T^{M} 1$ is a nonzero idempotent in $B$, $T E=E$ and $B=B E \oplus B(1-E)$ where $B E$ and $B(1-E)$ are invariant under $T$ and $T$ is nilpotent on $B(1-E)$. 
Proof. The lemma is trivial if $T 1=1$. Also if $T$ is nilpotent, then $T^{M} 1=0$ for some positive integer $M$ and there is nothing further to prove.

Assume $T$ is not nilpotent and $T 1 \neq 1$. Let $X$ denote the maximal ideal space of $B$ and $\varphi$ the continuous function on $X^{\prime}=$ $X \cup\{\theta\}$ induced by $T$. For each positive integer $n$, let $Z_{n}=\{x \in$ $\left.X \mid \varphi_{n}(x)=\theta\right\}$. (Since $\left.T 1 \neq 1, Z_{1} \neq \phi.\right)$ For each $n, Z_{n}$ is both open and closed in $X, \varphi^{-1}\left(Z_{n}\right)=Z_{n+1}$ and $Z_{n} \subset Z_{n+1}$. Also, $\varphi^{-1}\left(Z_{2} \backslash Z_{1}\right)=$ $\varphi^{-1}\left(Z_{2}\right)\left|\varphi^{-1}\left(Z_{1}\right)=Z_{3}\right| Z_{2}$ and, in general, $\varphi^{-n}\left(Z_{2} \mid Z_{1}\right)=Z_{n+2} \mid Z_{n+1}$ for each $n$.

We assert that $Z_{M}=Z_{M+1}$ for some positive integer $M$. To show this, assume $Z_{1} \neq Z_{2}$ and let $G$ be the element in $B$ such that $\hat{G}$ is the characteristic function of $Z_{2} \backslash Z_{1}$. Such an element exists by Silov's Idempotent Theorem $[1, \mathrm{p} .88]$ since $Z_{2} \backslash Z_{1}$ is a subset of $X$ which is both open and closed. By the definition of $G, \hat{G}(x)=1$ if $x \in Z_{2} \backslash Z_{1}$ and $\hat{G}(x)=0$ for all other $x \in X$; therefore for each positive integer $k, T^{k} G^{\wedge}(x)=G\left(\varphi_{k}(x)\right)=1$ if $x \in \varphi^{-k}\left(Z_{2} \mid Z_{1}\right)=Z_{k+2} \mid Z_{k+1}$ and $T^{k} G^{\wedge}(x)=0$ otherwise. We will now show that if $Z_{k+2} \mid Z_{k+1} \neq \phi$ for all positive integers $k$, then $\sigma(T) \supset\{\lambda|| \lambda \mid=1\}$ which will be a contradiction since $T$ is a compact operator. Thus assume $Z_{k+2} \mid Z_{k+1} \neq$ $\phi$ for all positive integers $k$ and choose $\lambda$ with $|\lambda|=1$. Let $n$ be a positive integer and consider $\left|\left[(\lambda+T)^{2 n} G\right]^{\wedge}(x)\right|$ for some $x \in Z_{n+2} \backslash Z_{n+1} \neq \phi$. Then

$$
\left|\left[(\lambda+T)^{2 n} G\right]^{\wedge}(x)\right|=\left|\left[\sum_{k=0}^{2 n} \lambda^{2 n+k}\left(\begin{array}{c}
2 n \\
k
\end{array}\right) T^{k} G\right]^{\wedge}(x)\right| .
$$

But if $x \in Z_{n+2} \mid Z_{n+1}$, then $T^{k} G^{\wedge}(x)=0$ unless $k=n$, and $\hat{G}\left(\varphi_{n}(x)\right)=$ $\left(T^{n} G\right)^{\wedge}(x)=1$. Therefore

$$
\left(\begin{array}{c}
2 n \\
n
\end{array}\right)=\left|\left(\begin{array}{c}
2 n \\
n
\end{array}\right) \hat{G}\left(\varphi_{n}(x)\right)\right|=\left|\left[(\lambda+T)^{2 n} G\right]^{\wedge}(x)\right| \leqq\left\|(\lambda+T)^{2 n}\right\|\|G\|
$$

and so

$$
\left(\begin{array}{c}
2 n \\
n
\end{array}\right)^{1 / 2 n} \leqq\left\|(\lambda+T)^{2 n}\right\|^{1 / 2 n}\|G\|^{1 / 2 n}
$$

If $Z_{n+2} \neq Z_{n+1}$ for all $n$, we can find such an $x$ for each positive integer $n$ and so $\left(^{*}\right)$ holds for all $n$. Also $\lim _{n \rightarrow \infty}\left(\begin{array}{l}2 n \\ n\end{array}\right)^{1 / 2 n}=2$. [2, Lemma 1.2]. Then letting $n \rightarrow \infty$ in (*) gives

$$
2=\lim _{n \rightarrow \infty}\left(\begin{array}{c}
2 n \\
n
\end{array}\right)^{1 / 2 n} \leqq \lim _{n \rightarrow \infty}\left\|(\lambda+T)^{2 n}\right\|\left\|^{1 / 2 n}=\right\| \lambda+T \|_{s p}
$$

for all $\lambda,|\lambda|=1$. However, from Lemma 1.1, $\|T\|_{s p}=1$. There- 
fore if $|\lambda|=1$, then $\lambda \in \sigma(T)$ and, as a result, every point in $\{\lambda|| \lambda \mid=1\}$ is in $\sigma(T)$, a contradiction. Therefore the assumption that $Z_{k+2} \mid Z_{k+1} \neq \phi$ for all positive integers $k$ is false and so there is a least positive integer $M$ for which $Z_{M}=Z_{M+1}$.

Now let $E=T^{M} 1$. Since $T$ is not nilpotent, $E$ is a nonzero idempotent in $B$. Also $\{x \in X \mid \hat{E}(x)=1\}=\left\{x \in X \mid\left(T^{M} 1\right)^{\wedge}(x)=1\right\}=$ $\left\{x \in X \mid \varphi_{M}(x) \neq \theta\right\}=X \backslash Z_{M}=X \backslash Z_{M+1}=\left\{x \in X \mid \varphi_{M+1}(x) \neq \theta\right\}=\{x \in$ $\left.X \mid\left(T^{M+1} 1\right)^{\wedge}(x)=1\right\}=\left\{x \in X \mid(T E)^{\wedge}(x)=1\right\}$. Therefore $T E=E$.

From Lemma 1.2, we have that $B E$ and $B(1-E)$ are commutative semi-simple Banach algebras which are invariant under $T$. The final assertion in the lemma that $T$ is nilpotent on $B(1-E)$ follows from the fact that $(1-E)$ is the multiplicative identity in $B(1-E)$ and $T^{M}(1-E)=T^{M} 1-T^{M} E=E-E=0$.

REMARK. Lemma 1.3 shows that $E=T^{M} 1$ is an eigenvector of $T$ in $B$ and so $1 \in \sigma(T)$ unless $T$ is nilpotent.

Next suppose $S$ is a nonempty closed subset of the maximal ideal space $X$ of a commutative semi-simple Banach algebra $B$ with unit 1. Then the kernel of $S, \operatorname{ker}(S)=\{f \in B \mid \hat{f}(t)=0$ for all $t \in S\}$ is a closed ideal in $B$ and $B_{1}=B / \operatorname{ker}(S)$ is a commutative semisimple Banach algebra with unit. If $X_{1}$ denotes the maximal ideal space of $B_{1}$, then $X_{1}$ is the hull of $\operatorname{ker}(S)$, i.e., $X_{1}=\{x \in X \mid f \in$ $\operatorname{ker}(S)$ implies $\hat{f}(x)=0\} . \quad X_{1}$ is a closed subset of $X$ and $S \subset X_{1} \subset X$. Further, if $x \in X_{1}$ and $\bar{f}=f+\operatorname{ker}(S) \in B / \operatorname{ker}(S)$, then $\bar{f}^{\wedge}(x)=\hat{f}(x)$ $[1$, p. 12].

Now let $T$ be an endomorphism of $B$ with $T 1=1$ and $\varphi$ the map of $X \rightarrow X$ induced by $T$. Clearly, if $\varphi(S) \subset S$, then $\operatorname{ker}(S)$ is invariant under $T$. Also if $\varphi(S) \subset S$, then $\varphi\left(X_{1}\right) \subset X_{1}$. For, if $\varphi(S) \subset S, f \in \operatorname{ker}(S)$ and $x \in X_{1}$, then $T f \in \operatorname{ker}(S)$, which implies $(T f)^{\wedge}(x)=0$ and this, in turn, implies $\hat{f}(\varphi(x))=0$, i.e., if $x \in X_{1}$, then $\varphi(x) \in X_{1}$. Thus $\varphi\left(X_{1}\right) \subset X_{1}$ if $\varphi(S) \subset S$.

Furthermore, if $\operatorname{ker}(S)$ is invariant under $T$, then $T$ induces an endomorphism $\bar{T}$ of $B_{1}$ into $B_{1}$ defined by $\bar{T} \bar{f}=\overline{T f}$ for $\bar{f} \in B_{1}$. Let $\bar{\varphi}$ be the map on $X_{1}$ induced by $\bar{T}$. Then by definition, $(\bar{T} \bar{f})^{\wedge}(x)=\bar{f}^{\wedge}(\bar{\varphi}(x))$ for all $x \in X_{1}$. We claim that $\bar{\varphi}=\left.\varphi\right|_{X_{1}}$. To this end, let $x \in X_{1}$. Then $\varphi(x) \in X_{1}$, and so $\bar{f}^{\wedge}(\bar{\varphi}(x))=(\bar{T} \bar{f})^{\wedge}(x)=(\overline{T f})^{\wedge}(x)=$ $(T f)^{\wedge}(x)=\hat{f}(\varphi(x))=\bar{f}^{\wedge}(\varphi(x))$. Since this holds for all $\bar{f} \in B_{1}$, it follows that $\bar{\varphi}(x)=\varphi(x)$ for each $x \in X_{1}$, as claimed. We remark, too, that if $T$ is a compact endomorphism, so is $\bar{T}$ [4].

With these observations we now prove the following.

Lemma 1.4. Assume $B$ is a commutative semi-simple Banach 
algebra with unit 1 and maximal ideal space $X$ and suppose $T$ is a compact endomorphism of $B$ with $T 1=1$. If $\varphi$ is the map on $X$ induced by $T$ and $\mathscr{S}=\cap \varphi_{n}(X)$, then $\mathscr{S}$ is a nonempty compact subset of $X$ and $\varphi$ maps $\mathscr{S}$ onto itself. Let $\operatorname{ker}(\mathscr{S})=\{f \mid \hat{f}(x)=0$ for all $x \in \mathscr{S}\}$ and set $B_{1}=B / \operatorname{ker}(\mathscr{S})$. Then $B_{1}$ is a commutative semi-simple Banach algebra with unit, and $X_{1}$, the maximal ideal space of $B_{1}$, satisfies $\mathscr{S} \subset X_{1} \subset X$. If $\bar{T}$ is defined on $B_{1}$ by $\bar{T} \bar{f}=$ $\overline{T f}$ for $\bar{f} \in B_{1}$, then $\bar{T}$ is a compact endomorphism of $B_{1}$ and $\sigma(\bar{T}) \subset$ $\{\lambda|| \lambda \mid=1\} \cup\{0\}$. Also, if $\bar{\varphi}$ is the map on $X_{1}$ induced by $\bar{T}$, then $\cap \bar{\varphi}_{n}\left(X_{1}\right)=\mathscr{S}$.

Proof. The properties of $B_{1}$ were discussed before the statement of the lemma. Also $\varphi$ maps $X$ into $X$ since $T 1=1$, and we have already noted in the introduction that $\phi$ maps $\mathscr{S}$ onto $\mathscr{S}$.

To prove that $\sigma(\bar{T}) \subset\{\lambda|| \lambda \mid=1\} \cup\{0\}$, suppose the contrary that there exists $\lambda, 0<|\lambda|<1, \lambda \in \sigma(\bar{T})$ and $\bar{T} \bar{f}=\lambda \bar{f}$. For each $x \in \mathscr{S}$ there are two possibilities.

(i) There exists a positive integer $N$ such that $\bar{\varphi}_{N}(x)=x$. In this case $\left(\bar{T}^{N} \bar{f}\right)^{\wedge}(x)=\bar{f}^{\wedge}\left(\bar{\varphi}_{N}(x)\right)=\bar{f}^{\wedge}(x)$ and also $\left(\bar{T}^{N} \bar{f}\right)^{\wedge}(x)=\lambda^{N} \bar{f}^{\wedge}(x)$. Therefore $\bar{f}^{\wedge}(x)=\lambda^{N} \bar{f}^{\wedge}(x)$, and since $|\lambda|<1, \bar{f}^{\wedge}(x)=0$.

(ii) For all $n, \bar{\varphi}_{n}(x) \neq x$. Since $\varphi$, and therefore $\bar{\varphi}$, maps $\mathscr{S}$ onto itself, we can choose distinct $t_{n} \in \mathscr{S}$ satisfying $\bar{\varphi}_{n}\left(t_{n}\right)=x$. Thus if $\bar{T} \bar{f}=\lambda \bar{f}$, then $\left(\bar{T}^{n} \bar{f}\right)^{\wedge}\left(t_{n}\right)=\bar{f}^{\wedge}\left(\varphi_{n}\left(t_{n}\right)\right)=\bar{f}^{\wedge}(x)$, while $\left(\bar{T}^{n} \bar{f}\right)^{\wedge}\left(t_{n}\right)=$ $\lambda^{n} \bar{f}^{\wedge}\left(t_{n}\right)$, also. Since $\left\|\bar{f}^{\wedge}\right\|_{\infty}<\infty$ and $\lambda^{n} \rightarrow 0$ it follows that $\bar{f}^{\wedge}(x)=$ $\lambda^{n} \bar{f}^{\wedge}\left(t_{n}\right) \rightarrow 0$. Hence if $\bar{\varphi}_{n}(x) \neq x$ for all $n$, then $\bar{f}^{\wedge}(x)=0$.

Thus we have just shown that if $0<|\lambda|<1$ and $\bar{T} \bar{f}=\lambda \bar{f}$, then $\bar{f}^{\wedge}(x)=0$ for all $x \in \mathscr{S}$. But this implies $\bar{f}=0$. Therefore all the nonzero eigenvalues of $\bar{T}$ lie on the unit circle.

All that remains to be shown is that $\mathscr{S}=\cap \bar{\varphi}_{n}\left(X_{1}\right)$. Now, $\cap \varphi_{n}\left(X_{1}\right)=\cap \bar{\varphi}_{n}\left(X_{1}\right)$ since $\bar{\varphi}=\left.\varphi\right|_{X_{1}}$. Therefore $\mathscr{S}=\cap \varphi_{n}(X) \supset$ $\cap \varphi_{n}\left(X_{1}\right)=\cap \bar{\varphi}_{n}\left(X_{1}\right) \supset \mathscr{S}$ which proves that $\mathscr{S}=\cap \bar{\varphi}_{n}\left(X_{1}\right)$.

Lemma 1.5. Suppose $B$ is a commutative semi-simple Banach algebra with unit 1 and maximal ideal space $X$. Let $T$ be a compact endomorphism of $B$ with $\sigma(T)=\{0,1\}$. Then there exists $a$ finite set of idempotents, $\left\{E_{1}, \cdots, E_{m}\right\}$, in $B$ with the following properties.

(i) $\left\{E_{1}, \cdots, E_{m}\right\}$ forms a basis for $\mathscr{N}=\{f \mid T f=f\}$ and $E_{i} E_{j}=\delta_{i j} E_{j}$.

(ii) If $E=\sum_{k=1}^{m} E_{k}$, then $B=B E_{1} \oplus \cdots \oplus B E_{m} \oplus B(1-E)$.

(iii) For each $k, k=1, \cdots, m, B E_{k}$ is a closed subalgebra of $B$ with multiplicative identity $E_{k}$. Also $B E_{k}$ is invariant under $T$ and all the eigenvectors of $T$ in $B E_{k}$ corresponding to 1 have the form $c E_{k}, c$ complex. 
(iv) If $E=\sum_{k=1}^{m} E_{k}$, then $B(1-E)$ is a closed subalgebra of $B$ with multiplicative identity $1-E . \quad B(1-E)$ is invariant under $T$ and $T$ is nilpotent on $B(1-E)$. Also $T^{M} 1=E$ for some positive integer $M$.

(v) If $T 1=1$, then $\sum_{k=1}^{m} E_{k}=1$ and $B=B E_{1} \oplus \cdots \oplus B E_{m}$.

Proof. (i ) Since $\sigma(T)=\{0,1\}, \mathscr{N}=\{f \mid T f=f\} \neq(0)$. Also $\mathscr{N}$ is closed under multiplication since $T(f g)=(T f)(T g)=f g$ whenever $f, g \in \mathscr{N}$. Further, since $T$ is a compact operator, $\mathscr{N}$ is finite dimensional. Therefore $\mathscr{N}$ is a finite dimensional commutative semi-simple Banach algebra and hence there exist idempotents $E_{1}, \cdots, E_{m}$ in $\mathscr{N}$ which form a basis for $\mathscr{N}$ and which satisfy $E_{i} E_{j}=\delta_{i j} E_{j}$. We note that since $E_{i} \in \mathscr{N}, i=1, \cdots, m, E_{i}$ must be an eigenvector of $T$ with $T E_{i}=E_{i}$.

(ii) Suppose $E=\sum_{k=1}^{m} E_{k}$. Then $1=\sum_{k=1}^{m} E_{k}+(1-E)$ and so for each $f \in B, f=\sum_{k=1}^{m} f E_{k}+f(1-E)$. Thus $B=B E_{1}+\cdots+$ $B E_{m}+B(1-E)$. Further, since $E_{i} E_{j}=\delta_{i j} E_{j}$ and $E_{i}(1-E)=0$ for all $i$, it is easy to verify that $f$ can be uniquely represented in this form. Therefore $B=B E_{1} \oplus \cdots \oplus B E_{m} \oplus B(1-E)$.

(iii) In view of Lemma 1.2 all that remains to be demonstrated here is that all the eigenvectors of $T$ in $B E_{j}$ corresponding to 1 have the form $c E_{j}, c$ complex. Now, if $T\left(f E_{j}\right)=f E_{j} \in B E_{j}$, then $f E_{j} \in \mathscr{N}$ so that $f E_{j}=\sum_{i=1}^{m} a_{i} E_{i}$. Therefore $f E_{j}=f E_{j}^{2}=\left(\sum_{i=1}^{m} a_{i} E_{i}\right) E_{j}=$ $a_{j} E_{j}$ as claimed.

(iv) $B(1-E)$ is a closed subalgebra of $B$ which is invariant under $T$ since $T E=E$. Also, since $\sigma(T)=\{0,1\}$, in order to prove that $T$ is nilpotent on $B(1-E)$, it suffices to show that $T(f(1-E))=$ $f(1-E)$ implies $f(1-E)=0$. But, if $T(f(1-E))=f(1-E)$, then $f(1-E) \in \mathscr{N} \cap B(1-E)=(0)$. Hence $T$ is nilpotent on $B(1-E)$ and so there exists a positive integer $M$ such that $T^{M}(1-E)=0$ or, equivalently, $T^{M} 1=T^{M} E=E$.

(v) If $T 1=1$, then $1=T^{M} 1=E$ from (iv). Therefore $1-E=0$ and $B=B E_{1} \oplus \cdots \oplus B E_{m}$.

Remark. The decomposition $B=B E_{1} \oplus \cdots \oplus B E_{m} \oplus B(1-E)$ leads to a splitting of the maximal ideal space $X$ of $B$ into disjoint open and closed subsets $Y_{1}, \cdots, Y_{m}, Y$, of $X$ where $Y_{k}=\left\{x \mid \hat{E}_{k}(x)=\right.$ $1\}, k=1, \cdots, m$ and $Y=X \backslash \bigcup_{k=1}^{m} Y_{k}$. Further, $Y_{k}$ is the maximal ideal space of $B E_{k}$ and $Y$ is the maximal ideal space of $B(1-E)$. If $\varphi$ is the map on $X \cup\{\theta\}$ induced by $T$, then $\varphi\left(Y_{k}\right) \subset Y_{k}, k=$ $1, \cdots, m$, and $\phi^{-1}\left(Y_{k}\right)=Y_{k}$. The last equality holds since $T E_{k}=E_{k}$.

The next lemma describes the behavior of $T^{*}$ on each $Y_{k}$. 
LeMma 1.6. Suppose $B$ is a commutative semi-simple Banach algebra with unit 1 and maximal ideal space $X$. Let $T$ be a compact endomorphism of $B$ with the property that $\sigma(T)=\{0,1\}$ and the only eigenvectors corresponding to 1 are the constants. If $\phi$ is the map on $X$ induced by $T$, then $\varphi$ maps $X$ into itself and there exists a unique element $\bar{x} \in X$ such that $\varphi(\bar{x})=\bar{x}$. Furthermore, $\lim _{n \rightarrow \infty} \hat{f}\left(\varphi_{n}(y)\right)=\hat{f}(\bar{x})$ for all $y \in X$ and $f \in B$, and $\cap \varphi_{n}(X)=\{\bar{x}\}$.

\section{Proof. The map $\varphi$ takes $X$ into itself since $T 1=1$.}

Since $T$ is a compact operator and the space of eigenvectors corresponding to 1 is one-dimensional by hypothesis, $B$ can be written $B=R_{1} \oplus N_{1}$ where $R_{1}=\{(T-I) f \mid f \in B\}$ and $N_{1}=\{f \mid T f=f\}$ $=(c)$. The closed subspaces $R_{1}$ and $N_{1}$ are invariant under $T$ [4].

Further, $T$ is quasinilpotent on $R_{1}$. For, if $g \in R_{1}$ and $T g=g$, then $g \in N_{1} \cap R_{1}=(0)$. Therefore 1 is not an eigenvalue of $T$ on $R_{1}$. Also there are no other eigenvalues of $T$ on $R_{1}$ since each eigenvalue of $T$ on $R_{1}$ is an eigenvalue of $T$ on $B$ and $\sigma(T)=\{0,1\}$ by hypothesis. Thus $T$ is quasinilpotent on $R_{1}$ and so

$$
\lim _{n \rightarrow \infty}\left(\sup _{T f \neq f} \frac{\left\|T^{n}(T-I) f\right\|}{\|(T-I) f\|}\right)^{1 / n}=\lim _{n \rightarrow \infty}\left\|T^{n}\right\|_{R_{1}}^{1 / n}=0 .
$$

Therefore for each $\varepsilon>0$ there exists $P^{*}>0$ such that $\left\|T^{n}(T-I) f\right\|<$ $P^{*} \varepsilon^{n}\|(T-I) f\|$ for all positive integers $n$ and all $f \in B$. Then letting $P=P^{*}\|T-I\|$ we have $\left\|T^{n}(T-I) f\right\|<P \varepsilon^{n}\|f\|$ for all positive integers $n$ and all $f \in B$.

Now fix $x \in X$. For each $f \in B,\left|\hat{f}\left(\varphi_{n+1}(x)\right)-\hat{f}\left(\varphi_{n}(x)\right)\right|=\mid[(T-$ $I) f]^{\wedge}\left(\Phi_{n}(x)\right)|=|\left[T^{n}(T-I) f\right]^{\wedge}(x) \mid \leqq\left\|T^{n}(T-I) f\right\|<P \varepsilon^{n}\|f\|$ for all positive integers $n$. Therefore $\left\{\hat{f}\left(\varphi_{n}(x)\right)\right\}$ is a Cauchy sequence of complex numbers and so $\lim _{n \rightarrow \infty} \hat{f}\left(\varphi_{n}(x)\right)$ exists for each $f \in B$. Let $l(f)=\lim _{n \rightarrow \infty} \hat{f}\left(\varphi_{n}(x)\right)$. Then it is easy to verify that $l$ is a linear multiplicative functional on $B$. Also $l \neq \theta$ since $T 1=1$ implies $l(1)=1 \neq 0$. Consequently there exists $\bar{x} \in X$ defined by $\hat{f}(\bar{x})=l(f)$ for all $f \in B$ and thus $\lim _{n \rightarrow \infty}\left(T^{n} f\right)^{\wedge}(x)=\lim _{n \rightarrow \infty} \hat{f}\left(\varphi_{n}(x)\right)=\hat{f}(\bar{x})$ for all $f \in B$. Also $T f \in B$, and so $\lim _{n \rightarrow \infty}(T f)^{\wedge}\left(\varphi_{n}(x)\right)=(T f)^{\wedge}(\bar{x})$ for all $f \in B$; this implies $\lim _{n \rightarrow \infty} \hat{f}\left(\varphi\left(\varphi_{n}(x)\right)=\hat{f}(\varphi(\bar{x}))\right.$. However,

$$
\lim _{n \rightarrow \infty} \hat{f}\left(\varphi\left(\varphi_{n}(x)\right)\right)=\lim _{n \rightarrow \infty} \hat{f}\left(\varphi_{n+1}(x)\right)=\hat{f}(\bar{x})
$$

for all $f \in B$. Therefore $\hat{f}(\varphi(\bar{x}))=\hat{f}(\bar{x})$ for all $f \in B$ which proves that $\bar{x}$ is a fixed point of $\varphi$.

We next show that $\cap \varphi_{n}(X)=\{\bar{x}\}$. To this end, let $M_{\bar{x}}=$ $\{f \mid \hat{f}(\bar{x})=0\}$. Since $\varphi(\bar{x})=\bar{x}$, the closed maximal ideal $M_{\bar{x}}$ is invariant under $T$. Also 1 is not an eigenvalue of $\left.T\right|_{M \bar{x}}$. For, if there exists $f \in M_{\bar{x}}$ with $T f=f$, then $f$ is an eigenvector of $T$ which must 
equal a constant $c$, say, by hypothesis. But $c=0$ since the only constant in $M_{\bar{x}}$ is 0 . Since $\sigma(T)=\{0,1\}, T$ is quasinilpotent on $M_{\bar{x}}$.

Now let $y$ be an arbitrary element in $X$. Since $f-\hat{f}(\bar{x}) 1 \in M \bar{x}$ and $T$ is quasinilpotent on $M \bar{x}$ we have that

$$
\lim _{n \rightarrow \infty}\left|\hat{f}\left(\varphi_{n}(y)\right)-\hat{f}(\bar{x})\right|^{1 / n}=\lim _{n \rightarrow \infty}\left|T^{n}(f-\hat{f}(\bar{x}) 1)^{\wedge}(y)\right|^{1 / n}=0 .
$$

Using an argument similar to one used in the first part of this proof, it can be shown that for each $\varepsilon>0$ there exists $P_{1}>0$ such that $\left|\hat{f}\left(\varphi_{n}(y)\right)-\hat{f}(\bar{x})\right|<P_{1} \varepsilon^{n}\|f\|$ for all $f \in B, n>0$ and $y \in X$. This implies that if $\mathscr{W}$ is an open subset of $X$ with $\bar{x} \in \mathscr{U}$, then $\varphi_{n}(X) \subset \mathscr{U}$ for large $n$. Therefore $\cap \varphi_{n}(X)=\{\bar{x}\}$. It now follows easily that $\bar{x}$ is the only fixed point of $\varphi$.

(The uniqueness of $\bar{x}$ also follows from the fact that the dimensions of $\{f \mid T f=f\}$ and $\left\{l \in B^{*} \mid T^{*} l=l\right\}$ are equal. Since $\{f \mid T f=f\}$ is one dimensional, once we have shown that $\bar{x}$ is a fixed point of $\varphi$ in $X$, then it must be unique.)

We now combine these lemmas to prove the following.

THeOREM 1.7. Suppose $B$ is a commutative semi-simple Banach algebra with unit 1 and maximal ideal space $X$ and $T$ is a nonzero compact endomorphism of $B$. If $\varphi$ is the map on $X^{\prime}=X \cup\{\theta\}$ induced by $T$, then $\cap \varphi_{n}\left(X^{\prime}\right)$ is finite. If $X$ is connected, then $\cap \varphi_{n}(X)$ is a singleton.

Proof. If $T$ is nilpotent, then $\cap \varphi_{n}\left(X^{\prime}\right)=\{\theta\}$ and there is nothing further to prove.

Assume $T$ is not nilpotent. From Lemma 1.3 there exists a smallest positive integer $M$ and a nonzero idempotent $E=T^{M} 1$ with the property that $T E=E, T: B E \rightarrow B E$ and $B=B E \oplus B(1-E)$. Also $Z=\{x \in X \mid \hat{E}(x)=1\}$ is the maximal ideal space of $B E, \varphi(Z) \subset Z$ and $\varphi_{M}: X \backslash Z \rightarrow\{\theta\}$. Let $\mathscr{S}=\cap \varphi_{n}(Z)$. Since $\cap \varphi_{n}\left(X^{\prime}\right)=\mathscr{S} \cup\{\theta\}$ it suffices to prove that $\mathscr{S}$ is finite.

Consider $T$ on $B E$. Since $E$ is a unit in $B E$ and $T E=E$, Lemma 1.4 implies that $T$ induces a compact endomorphism $\bar{T}$ on $B_{1}=B E / \operatorname{ker}(\mathscr{S})$ which satisfies $\bar{T} \bar{E}=\bar{E}$ and $\sigma(\bar{T}) \subset\{\lambda|| \lambda \mid=1\} \cup$ $\{0\}$. Letting $X_{1}$ denote the maximal ideal space of $B_{1}$ and $\bar{\varphi}$ the map on $X_{1}$ induced by $\bar{T}$, Lemma 1.4 also implies $\mathscr{S}=\cap \bar{\varphi}_{n}\left(X_{1}\right)$.

Since $\bar{T}$ is a compact endomorphism on $B_{1}$ and $\sigma(\bar{T}) \subset\{\lambda|| \lambda \mid=1\}$ $\cup\{0\}$, each nonzero eigenvalue of $\bar{T}$ is a root of unity and so there exists a positive integer $N$ for which $\sigma\left(\bar{T}^{N}\right)=\{0,1\}$. Also $\bar{T} \bar{E}=\bar{E}$ implies $\bar{T}^{N} \bar{E}=\bar{E}$. Therefore $\bar{T}^{N}$ is a compact endomorphism of $B_{1}$ with $\sigma\left(\bar{T}^{N}\right)=\{0,1\}$ and by Lemma $1.5, B_{1}$ can be written $B_{1}=B_{1} \bar{E}_{1} \oplus \cdots \oplus B_{1} \bar{E}_{m}$ where $\bar{E}=\sum_{k=1}^{m} \bar{E}_{k}, \bar{E}_{k}$ are idempo- 
tents in $B_{1}, \bar{T}^{N} \bar{E}_{k}=\bar{E}_{k}$ and all the eigenvectors of $\bar{T}^{N}$ on $B_{1} \bar{E}_{k}$ corresponding to 1 have the form $c \bar{E}_{k}$, $c$ complex. We also have that $X_{1}=Y_{1} \cup \cdots \cup Y_{m}$ where $Y_{k}$ is the maximal ideal space of $B_{1} \bar{E}_{k}$. It is clear that $\bar{\varphi}_{N}$ is the map on $X_{1}$ induced by $\bar{T}^{N}$ and so we have that $\bar{\varphi}_{N}\left(Y_{k}\right) \subset Y_{k}, k=1, \cdots, m$. Thus $\cap \bar{\varphi}_{N n}\left(X_{1}\right)=$ $\cap \bar{\varphi}_{N n}\left(Y_{1}\right) \cup \cdots \cup \cap \bar{\varphi}_{N n}\left(Y_{m}\right)$.

Now using the fact that all the eigenvectors of $\bar{T}^{N}$ on $B_{1} \bar{E}_{k}$ have the form $c \bar{E}_{k}$, $c$ complex, it follows from Lemma 1.6 that there exist $\bar{x}_{k} \in Y_{k}$ with $\cap \bar{\varphi}_{N n}\left(Y_{k}\right)=\left\{\bar{x}_{k}\right\}, k=1, \cdots, m$. Therefore

$$
\begin{aligned}
\mathscr{S}=\cap \varphi_{n}(Z) & =\cap \bar{\varphi}_{n}\left(X_{1}\right)=\cap \bar{\varphi}_{N n}\left(X_{1}\right) \\
& =\cap \bar{\varphi}_{N n}\left(Y_{1}\right) \cup \cdots \cup \cap \bar{\varphi}_{N n}\left(Y_{m}\right) \\
& =\left\{\bar{x}_{1}, \cdots, \bar{x}_{m}\right\} .
\end{aligned}
$$

Thus $\mathscr{S}$ is finite and hence $\cap \varphi_{n}\left(X^{\prime}\right)$ is finite, as needed.

Finally, if $X$ is connected, then the only nonzero idempotent in $B$ is 1 . In this case $T 1=1$ and therefore $\varphi$ maps $X$ into itself. Hence $S=\cap \varphi_{n}(X)$ is connected and since $S$ is finite, $S$ must be a singleton.

2. We conclude with several miscellaneous theorems and examples relating to compact endomorphisms.

It was noted in the introduction that if $a$ is a specific point in the maximal ideal space of a commutative semi-simple Banach algebra with unit 1 , then the map $T: f \rightarrow \widehat{f}(a) 1$ is a compact endomorphism of $B$. We will show that if $X$ is a compact connected Hausdorff space, then every nonzero compact endomorphism of $C(X)$ has this form. We also show that the same is true for $C^{1}$, the algebra of functions on $[0,1]$ with continuous first derivatives. We will begin this section with a theorem about compact endomorphisms of function algebras.

Recall that a function algebra is a sup-norm closed subalgebra of continuous functions on a compact set $X$ which separates points of $X$ and contains the constants. A peak set of a function algebra is a closed subset $E$ of $X$ for which there exists a function $f$ in the algebra with $\|f\|=f(x)=1$ for $x \in E$ and $|f(x)|<1$ for $x \in$ $X \backslash E$. A generalized peak point is a point $x_{0}$ in $X$ such that $\left\{x_{0}\right\}$ is an intersection of peak sets, and the strong boundary of a function algebra is the collection of generalized peak points. Further, if $W$ is a $G_{o}$ subset of $X$ containing a generalized peak point $x_{0}$, then there exists a peak set $E$ with $x_{0} \in E \subset W$ [1].

THEOREM 2.1. Let $X$ be a compact connected Hausdorff space and suppose $B$ is a function algebra on $X$ whose maximal ideal 
space is $X$. Further, assume $0 \neq T$ is a compact endomorphism of $B$ with $\varphi$ the continuous function on $X$ induced by $T$. If $\varphi\left(x_{0}\right)$ is a generalized peak point of $B$ for some $x_{0} \in X$, then $T f=f\left(\varphi\left(x_{0}\right)\right) 1$ for all $f \in B$.

Proof. Assume $\varphi\left(x_{0}\right)$ is a generalized peak point of $B$. The claim is that $\varphi(x)=\varphi\left(x_{0}\right)$ for all $x \in X$. Suppose the contrary that there exists $y \in X$ with $\varphi(y) \neq \varphi\left(x_{0}\right)$. Since $\varphi\left(x_{0}\right)$ is a generalized peak point, there exists a peak set $E$ such that $\varphi\left(x_{0}\right) \in E$ and $\varphi(y) \notin$ $E$. For this set $E$, let $f \in B$ satisfy $\|f\|=f(x)=1$ for all $x \in E$ and $|f(x)|<1$ for $x \in X \backslash E$. Further, let $f_{n}=\left(\frac{1}{2}(1+f)\right)^{n}$. Then $\left\|f_{n}\right\|=$ 1 and since $T$ is a compact operator, there exist a subsequence $\left\{f_{n_{k}}\right\}$ and a function $g \in B$ with $T f_{n_{k}} \rightarrow g$ uniformly. Clearly $\lim _{n \rightarrow \infty}\left(\frac{1}{2}(1+\right.$ $f(x)))^{n}=1$ if $f(x)=1$ and $\lim _{n \rightarrow \infty}\left(\frac{1}{2}(1+f(x))\right)^{n}=0$ if $f(x) \neq 1$. Since $g(x)=\lim _{k \rightarrow \infty}\left(\frac{1}{2}(1+f(\varphi(x)))\right)^{n_{k}}$ for $x \in X$, the continuous function $g$ can assume at most two values, 0 and 1 . However, the domain of $g$ is connected. Hence $g$ must be constant. This leads to a contradiction since if $\varphi(y) \notin E$, then $g(y)=\lim _{k \rightarrow \infty}\left(\frac{1}{2}(1+f(\varphi(y)))\right)^{n_{k}}=0$ while $g\left(x_{0}\right)=\lim _{k \rightarrow \infty}\left(\frac{1}{2}\left(1+f\left(\varphi\left(x_{0}\right)\right)\right)\right)^{n_{k}}=1$. Therefore $\varphi(x)=\varphi\left(x_{0}\right)$ for all $x \in X$ as claimed.

If $X$ is a compact Hausdorff space, then every $x \in X$ is a generalized peak point of $C(X)$. Consequently, we have the following immediate corollary of Theorem 2.1.

COROLLARY 2.2. If $X$ is a compact connected Hausdorff space, then every nonzero compact endomorphism $T$ of $C(X)$ has the form $T f=f\left(x_{0}\right) 1$ for some $x_{0} \in X$.

THEOREM 2.3. Let $C^{1}$ be the algebra of functions on $[0,1]$ with continuous first derivatives, pointwise arithmetic operations and $\|f\|=\|f\|_{\infty}+\left\|f^{\prime}\right\|_{\infty}$. Then every nonzero compact endomorphism $T$ on $C^{1}$ has the form $T f=f(c) 1$ for some $c \in[0,1]$.

Proof. Let $T$ be a compact endomorphism of $C^{1}$ and $\varphi$ the map on $[0,1]$ induced by $T$. Then $\phi \in C^{1}$. We claim that $\varphi$ is a constant function. Suppose $\varphi$ is not constant. Then there exists $a \in(0,1)$ with $\varphi^{\prime}(a) \neq 0$. Let $b=\varphi(a)$. Then $b \in(0,1)$. For each positive integer $n$, let $f_{n}(x)=\int_{0}^{x} e^{-n(b-t)^{2}} d t$. Then $f_{n} \in C^{1}$, $\sup _{0 \leqq x \leqq 1}\left|f_{n}(x)\right|=$ $\int_{0}^{1} e^{-n(b-t)^{2}} d t<1$ and $\sup _{0 \leqq x \leqq 1}\left|f_{0}^{\prime}(x)\right|=\sup _{0 \leqq x \leqq 1} e^{-n(b-x)^{2}}=1$. Therefore $\left\|f_{n}\right\|<2$ for all $n$. Since $\left\{f_{n}\right\}$ is a bounded set in $C^{1}$ and $T$ is a compact endomorphism, there exist $g \in C^{1}$ and $\left\{f_{n_{k}}\right\}$ with $T f_{n_{k}} \rightarrow g$. In particular $g^{\prime}$ is continuous and $\left(T f_{n_{k}}\right)^{\prime} \rightarrow g^{\prime}$ uniformly. Now 


$$
\begin{array}{r}
\left(T f_{n_{k}}\right)^{\prime}(x)=f_{n_{k}}{ }^{\prime}(\varphi(x)) \phi^{\prime}(x)=e^{-n_{k}(b-\varphi(x))^{2}} \phi^{\prime}(x), \text { and hence } \\
g^{\prime}(a)=\lim _{k \rightarrow \infty} e^{-n_{k}(b-\varphi(a))^{2}} \phi^{\prime}(a)=\varphi^{\prime}(a) .
\end{array}
$$

Since $\varphi(a)=b, g^{\prime}(a) \neq 0$. However, since $\varphi^{\prime}(a) \neq 0, \varphi(x) \neq b$ in some deleted interval about $a$, and so it follows that

$$
g^{\prime}(x)=\lim _{k \rightarrow \infty} e^{-n_{k}(b-\varphi(x))^{2}} \varphi^{\prime}(x)=0
$$

in that deleted interval. This is a contradiction to the continuity of $g^{\prime}$. Hence $\varphi^{\prime}=0$ and $\varphi$ is a constant function. Therefore $(T f)(x)=f(\varphi(x))=f(c)$ for some $c \in[0,1]$ and so $T f=f(c) 1$.

Modifications of the statements and proofs of Theorem 2.1 and Corollary 2.2 for disconnected $X$ are straightforward. For example, if $X$ is an arbitrary compact Hausdorff space and $T$ is a compact endomorphism of $C(X)$, then there exist a finite number of idempotents $E_{1}, \cdots, E_{m}$ in $C(X)$ and points $t_{1}, \cdots, t_{m} \in X$ with $T f=$ $\sum_{k=1}^{m} f\left(t_{k}\right) E_{\pi(k)}$ where $\pi$ is a permutation of the set of integers $\{1, \cdots, m\}$.

There is a similarity between Theorem 2.1 and the example of the disc algebra, namely, that in both cases the range of a nonconstant $\varphi$ does not intersect the strong boundary. However, it is not possible to extend this by replacing strong boundary with Silov boundary as the following example shows. ( $\boldsymbol{C}$ and $\boldsymbol{R}$ denote the complex and real numbers, respectively.)

Example. Let $X$ be the subset of $\boldsymbol{C} \times \boldsymbol{R}$ defined by $X=$ $\{(z, 0)|| z \mid \leqq 1\} \cup\{(0, t) \mid 0 \leqq t \leqq 1\}$ and let $B=\{f \in C(X) \mid z \rightarrow f(z, 0)$ is analytic\}. Then $B$ is a function algebra whose Silov boundary is $\{(z, 0)|| z \mid=1\} \cup\{(0, t) \mid 0 \leqq t \leqq 1\}$. The point $(0,0)$ is in the Silov boundary, but is not a generalized peak point. Define $\varphi$ on $X$ by $\varphi(z, 0)=(z / 2,0)$ and $\varphi(0, t)=(0,0)$. Then it is easy to verify that $T: T f=f \circ \varphi$ is a compact endomorphism of $B$ and $\varphi(0,0)=(0,0)$ is in the Silov boundary. However $T$ does not have the form $T f=$ $f(0,0) 1$. Note, though, that $\cap \varphi_{n}(X)=\{(0,0)\}$.

Another reasonable conjecture from the example of the disc algebra might be that if $T$ is an endomorphism of a function algebra $B$ on $X$ for which $\varphi(X)$ does not intersect the Silov boundary, then $T$ is compact. This, too, is not true.

ExAmple. Let $X=\{(z, t)|| z \mid \leqq 1$ and $0 \leqq t \leqq 1\}$ and let $B=$ $\{f \in C(X) \mid z \rightarrow f(z, t)$ is analytic for each $t\}$. The Silov boundary of $B$ is $\{(z, t)|| z \mid=1,0 \leqq t \leqq 1\}$. Define $\varphi$ by $\varphi(z, t)=(z / 2, t)$. Then 
$\varphi(X)=\{(z, t)|| z \mid \leqq 1 / 2,0 \leqq t \leqq 1\}$ does not intersect the Silov boundary, yet $T f=f \circ \varphi$ is not compact since, for instance, $\cap \varphi_{n}(X)=$ $\{(0, t) \mid 0 \leqq t \leqq 1\}$ is not a singleton.

As a final example along these lines, we note that even if $\cap \varphi_{n}(X)$ is a singleton, the endomorphism $T f=f \circ \varphi$ need not be compact. For, let $B=C(\bar{D})$, the algebra of continuous functions on the closed unit disc $\bar{D}$ and let $\varphi(z)=z / 2$. Then $\cap \varphi_{n}(\bar{D})=\{0\}$, while $T f=f \circ \varphi$ is not compact because, as we have seen, each compact endomorphism on $C(\bar{D})$ has the form $T f=f(a) 1$ for some $a \in \bar{D}$.

\section{REFERENCES}

1. T.W. Gamelin, Uniform Algebras, Prentice-Hall, Inc., Englewood Cliffs, N.J., 1969.

2. H. Kamowitz, The spectra of a class of operators on the disc algebra, Indiana Univ. Math. J., 27 (1978), 581-610.

3. Compact operators of the form $u C_{\varphi}$, Pacific J. Math., 80 (1979), 205-211.

4. J. R. Ringrose, Compact Non-Self-Adjoint Operators, Van Nostrand Reinhold Co., London, 1971, 50-51.

Received September 12, 1979 and in revised form November 29, 1979.

University of Massachusetts/Boston

Dorchester, MA 02125 



\section{PACIFIC JOURNAL OF MATHEMATICS}

\section{EDITORS}

DONALD BABBITT (Managing Editor)

University of California

Los Angeles, CA 90024

HUGo RossI

University of Utah

Salt Lake City, UT 84112

C. C. MOORE and ANDREW OGG

University of California

Berkeley, CA 94720
J. DugundJI

Department of Mathematics

University of Southern California

Los Angeles, CA 90007

R. FinN and J. Milgram

Stanford University

Stanford, CA 94305

ASSOCIATE EDITORS
E. F. BECKENBACH
B. H. NeUmanN
F. WOLF
K. YoSHIDA

\section{SUPPORTING INSTITUTIONS}

UNIVERSITY OF BRITISH COLUMBIA

CALIFORNIA INSTITUTE OF TECHNOLOGY

UNIVERSITY OF CALIFORNIA

MONTANA STATE UNIVERSITY

UNIVERSITY OF NEVADA, RENO

NEW MEXICO STATE UNIVERSITY

OREGON STATE UNIVERSITY

UNIVERSITY OF OREGON
UNIVERSITY OF SOUTHERN CALIFORNIA

STANFORD UNIVERSITY

UNIVERSITY OF HAWAII

UNIVERSITY OF TOKYO

UNIVERSITY OF UTAH

WASHINGTON STATE UNIVERSITY

UNIVERSITY OF WASHINGTON

The Supporting Institutions listed above contribute to the cost of publication of this Journal, but they are not owners or publishers and have no responsibility for its content or policies.

Mathematical papers intended for publication in the Pacific Journal of Mathematics should be in typed form or offset-reproduced, (not dittoed), double spaced with large margins. Please do not use built up fractions in the text of the manuscript. However, you may use them in the displayed equations. Underline Greek letters in red, German in green, and script in blue. The first paragraph or two must be capable of being used separately as a synopsis of the entire paper. Please propose a heading for the odd numbered pages of less than 35 characters. Manuscripts, in triplicate, may be sent to any one of the editors. Please classify according to the scheme of Math. Reviews, Index to Vol. 39. Supply name and address of author to whom proofs should be sent. All other communications should be addressed to the managing editor, or Elaine Barth, University of California, Los Angeles, California, 90024.

50 reprints to each author are provided free for each article, only if page charges have been substantially paid. Additional copies may be obtained at cost in multiples of 50 .

The Pacific Journal of Mathematics is issued monthly as of January 1966. Regular subscription rate: $\$ 84.00$ a year (6 Vols., 12 issues). Special rato: $\$ 42.00$ a year to individual members of supporting institutions.

Subscriptions, orders for numbers issued in the last three calendar years, and changes of address shoud be sent to Pacific Journal of Mathematics, P.O. Box 969, Carmel Valley, CA 93924, U.S.A Old back numbers obtainable from Kraus Periodicals Co., Route 100, Millwood, NY 10546.

PUBLISHED BY PACIFIC JOURNAL OF MATHEMATICS, A NON-PROFIT CORPORATION

Printed at Kokusai Bunken Insatsusha (International Academic Printing Co., Ltd.). 8-8, 3-chome, Takadanobaba, Shinjuku-ku, Tokyo 160, Japan.

Copyright (C) 1980 by Pacific Jounal of Mathematics Manufactured and first issued in Japan 


\section{Pacific Journal of Mathematics \\ Vol. 89, No. $2 \quad$ June, 1980}

Frank Hayne Beatrous, Jr. and R. Michael Range, On holomorphic

approximation in weakly pseudoconvex domains................. 249

Lawrence Victor Berman, Quadratic forms and power series fields ...... 257

John Bligh Conway and Wacław Szymański, Singly generated antisymmetric operator algebras ....................... 269

Patrick C. Endicott and J. Wolfgang Smith, A homology spectral sequence for submersions . . . .................................

Sushil Jajodia, Homotopy classification of lens spaces for one-relator groups with torsion ................................ 301

Herbert Meyer Kamowitz, Compact endomorphisms of Banach

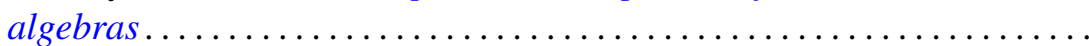

Keith Milo Kendig, Moiré phenomena in algebraic geometry: polynomial

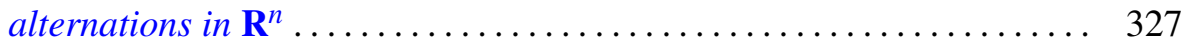

Cecelia Laurie, Invariant subspace lattices and compact operators....... 351

Ronald Leslie Lipsman, Restrictions of principal series to a real form . . . . . 367

Douglas C. McMahon and Louis Jack Nachman, An intrinsic

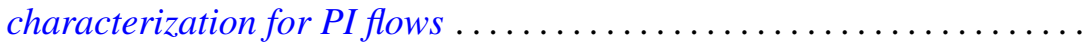

Norman R. Reilly, Modular sublattices of the lattice of varieties of inverse semigroups .................................... 405

Jeffrey Arthur Rosoff, Effective divisor classes and blowings-up of $\mathbf{P}^{2}$ 419

Zalman Rubinstein, Solution of the middle coefficient problem for certain

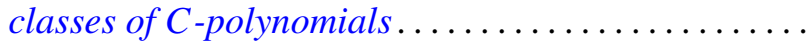

Alladi Sitaram, An analogue of the Wiener-Tauberian theorem for spherical transforms on semisimple Lie groups ................

Hal Leslie Smith, A note on disconjugacy for second order systems ...

J. Wolfgang Smith, Fiber homology and orientability of maps ...

Audrey Anne Terras, Integral formulas and integral tests for series of positive matrices. 